\title{
Transient microscopic testing method based on deflectometry
}

Gu, Hanting, Wang, Daodang, Xie, Zhongming, Kong, Ming, Liang, Rongguang, et al.

Hanting Gu, Daodang Wang, Zhongming Xie, Ming Kong, Rongguang Liang, Wentao Zhang, "Transient microscopic testing method based on deflectometry," Proc. SPIE 11185, Optical Design and Testing IX, 111850R (19 November 2019); doi: 10.1117/12.2537149

SPIE. Event: SPIE/COS Photonics Asia, 2019, Hangzhou, China 


\title{
Transient microscopic testing method based on deflectometry
}

\author{
Hanting Gu ${ }^{\mathrm{a}}$, Daodang Wang ${ }^{* a}$, Zhongmin Xie ${ }^{\mathrm{a}}$, Ming Kong ${ }^{\mathrm{a}}$, Rongguang Liang ${ }^{\mathrm{b}}$, Wentao Zhang ${ }^{\mathrm{c}}$ \\ ${ }^{a}$ College of Metrology and Measurement Engineering, China Jiliang University, Hangzhou 310018, \\ China; ${ }^{b}$ College of Optical Sciences, University of Arizona, Tucson, Arizona 85721, USA; ${ }^{\mathrm{c}}$ Guangxi \\ Key Laboratory of Optoelectronic Information Processing (Guilin University of Electronic \\ Technology), Guilin 541004, China
}

\begin{abstract}
The deflectometry provides an optical testing method with ultra-high dynamic range. In this paper, a microscopic testing method based on deflectometric technique is proposed to quantitatively evaluate the microstructures according to the wavefront aberration. To achieve the real-time and accurate wavefront testing for microstructure evaluation, a colorcoded phase-shifting fringe pattern is applied to illuminate the test object. It avoids the sequential projection of multistep phase-shifting fringes in traditional deflectometry, enabling the transient wavefront testing. The feasibility of the proposed transient microscopic testing method is demonstrated by the experiment. The proposed method enables accurate and transient testing of microstructures with high dynamic range, minimizing the environmental disturbance.
\end{abstract}

Keywords: microscopic deflectometry; wavefront aberration; transient test; large dynamic range

\section{INTRODUCTION}

With the rapid development of optical fabrication, the measurement of surface shape and roughness is required in many fields, including precision engineering [1], micro-manufacturing [2], quality testing and biotechnology [3], etc. Either the contact or non-contact methods can be applied to measure the microscopic surface information. The stylus profiler, as a typical contact method, has a large dynamic range and can achieve accurate measurement. However, it is easy to damage the workpiece in the testing process. Optical profilers and interference microscopes provide feasible and noncontact ways to achieve microscopic measurement. The point-to-point sampling data in profilers $[4,5]$ needs to be spliced to obtain the full-aperture surface; besides, the measurement with the profiler is inefficient, time-consuming and can be easily affected by scanning mechanism precision and environmental disturbance. The interference microscope [6-9] has been serving as an accurate, highly efficient way for the roughness measurement. However, its dynamic range is quite limited, making it not suitable for the testing of surfaces with large slopes.

As a slope measuring technique, the deflectometry [10-14] enables the surface testing with large dynamic range and extremely simple system configuration. The deflectometric testing system generally consists of an LCD projection screen, camera and test object. Using the sinusoidal fringe projection, the surface slopes can be calculated with phaseshifting techniques. It provides a simple and low-cost testing method with ultra-large dynamic range. The deflectometry has been successfully applied to test the spherical mirrors, aspheric mirrors and precision X-ray mirrors, etc., with the accuracy comparable with the interferometry. Besides, various micro-deflectometric systems have also been proposed for the microscopic measurement [15-18], which are traditionally based on phase-shifting fringe projection method. The phase-shifting method is time consuming and not suitable for transient measurement.

In this paper, a transient microscopic testing method based on deflectometry is proposed to quantitatively evaluate the microstructures according to the wavefront aberration. In the deflectometry-based microscopic testing, the illumination module shares the same optical circuit with the detection path through a microscope objective. In addition, the multi-step phase-shifting fringes for illumination are encoded in the color channels to achieve transient testing with a single shot. Section 2 presents the principle of transient microscopic testing method. In Section 3, the experimental result is given to demonstrate the feasibility of the proposed method. In addition, some conclusions are drawn in Section 4.

*wangdaodang@sina.com; phone 86-571-86914563

Optical Design and Testing IX, edited by Yongtian Wang, Pablo Benítez,

Osamu Matoba, Proc. of SPIE Vol. 11185, 111850R - (C) 2019 SPIE

CCC code: $0277-786 X / 19 / \$ 21 \cdot$ doi: $10.1117 / 12.2537149$ 


\section{PRINCIPLE}

\subsection{Principle of microscopic testing method based on deflectometry}

Figure 1 shows the schematic diagram of microscopic testing system based on deflectometry, which consists of the illumination and detection paths. The fringe pattern from illumination screen is reflected by a beam splitter and imaged on the plane in front of test object by a microscope objective. The light reflected from test object is collected by the microscope objective, and then passes through the beam splitter, finally is captured by the CCD camera.

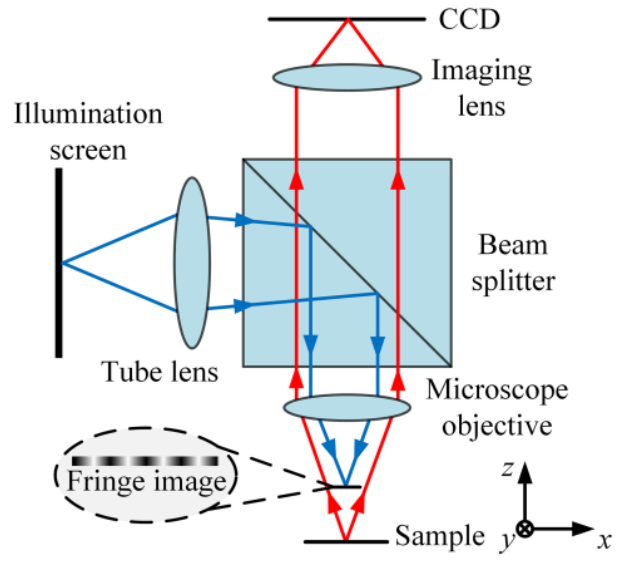

Fig. 1. The diagram of the microscopic testing system based on deflectometry.

Based on four-step phase-shifting algorithm, the observed phase $\left(\varphi_{x}, \varphi_{y}\right)$ can be calculated according to the acquired illumination fringes. Without loss of generality, we take the slopes in $x z$ plane as the case for analysis, as is shown in Fig. 2. According to Fig. 2, the relationship between the observed phase $\varphi_{x}$ and the surface slope $k_{x}$ at the point M can be described as

$$
k_{x}=\frac{p}{2 \pi d} \varphi_{x},
$$

where $d$ is the distance between the image plane of projected fringe and test object, and $p$ is the fringe period. Based on the sinusoidal fringe illumination and phase-shifting method, the surface slopes $\left(k_{x}, k_{y}\right)$ in $x$ and $y$ directions can be measured from the observed phases $\left(\varphi_{x}, \varphi_{y}\right)$, by which the test surface can be reconstructed with integration method.

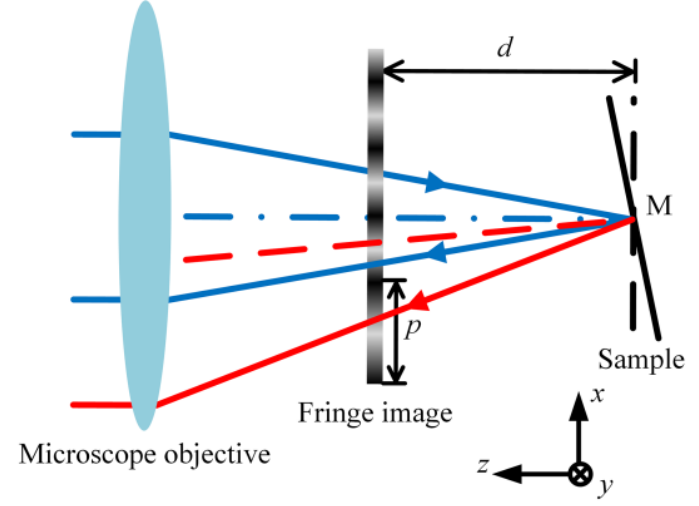

Fig. 2. The general relationship between observed phase and surface slopes.

\subsection{Transient wavefront testing method}

Figure 3 shows the whole procedure for the fringe pattern processing in the proposed instantaneous testing method. The original pattern for projection is a color-coded composite pattern, in which the multiple phase-shifting fringes in $x$ and $y$ directions are integrated in a single-color image. With the instantaneously acquired distorted composite image, the color 
separation is firstly applied to extract the composite fringe pattern in $x$ and $y$ directions from the red and blue channels, respectively. In each separated pattern, the four-step phase-shifted fringe information is included in the distinctly designed frequency carriers in each direction. To obtain the four-individual phase-shifted fringe patterns in $x$ and $y$ directions, a demodulation method based on Butterworth filter [19] is designed. Using the extracted four-step phase shift fringes in $x$ and $y$ directions, the corresponding observed phase distributions $\left(\varphi_{x}, \varphi_{y}\right)$ can be obtained for the further deflectometric wavefront measurement [20]. The local surface slopes in the $x$ and $y$ directions can be simultaneously calculated with the extracted multi-step phase-shifting fringe patterns, in which only a single shot is required for the information extraction for wavefront reconstruction, and it provides a feasible way for the transient testing. In this proposed transient wavefront testing method, the crosstalk among RGB channels and photon response nonuniformity could be largely avoided, and it does not require complex calibration.

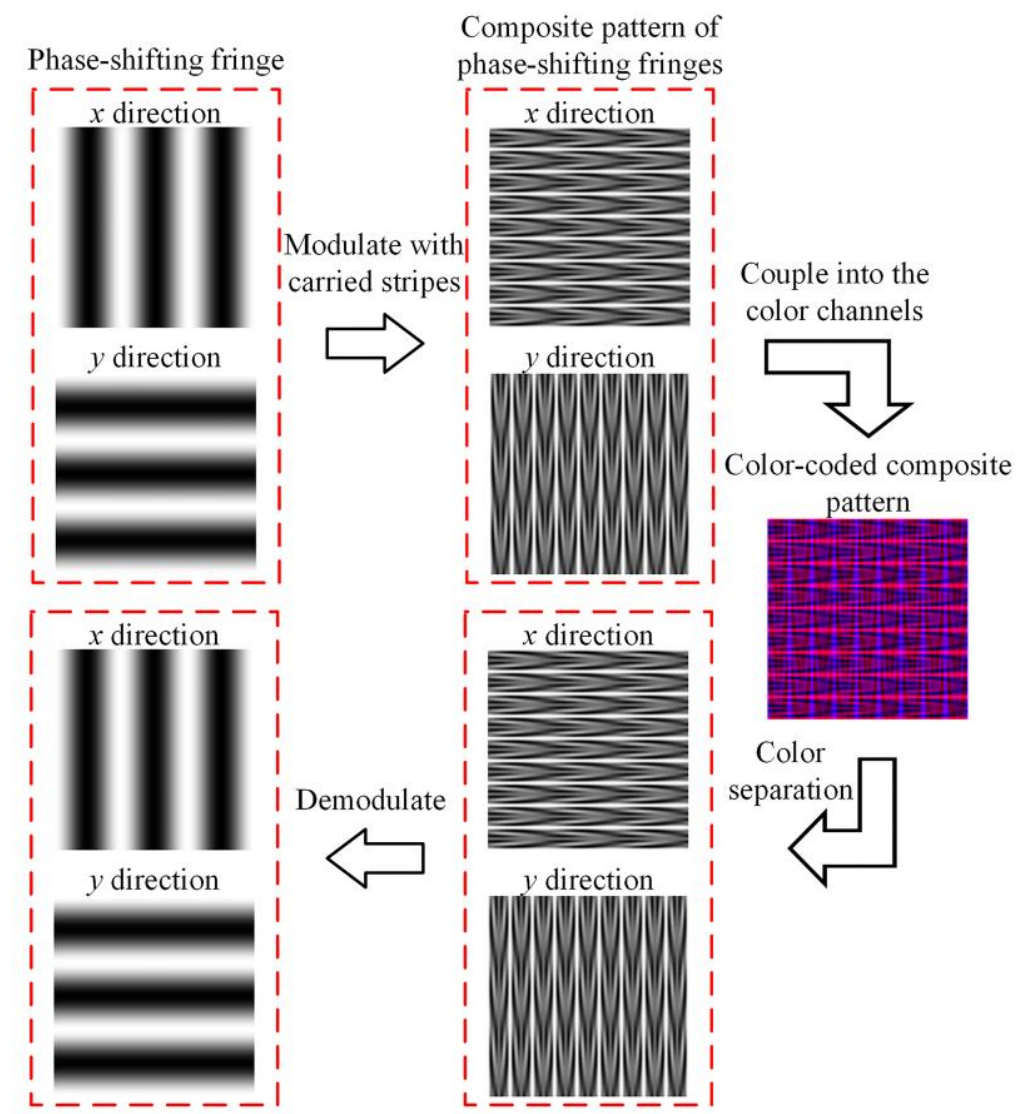

Fig. 3. Extraction of multi-step phase-shifting fringe in the proposed color-coded composite pattern for transient testing.

\section{EXPERIMENT RESULTS}

According to Fig. 1, a microscopic testing system based on deflectometry has been built to demonstrate the feasibility of the proposed method for microscopic testing. The pixel number of LCD projection screen is $3840(\mathrm{H}) \times 2160(\mathrm{~V})$, with the corresponding pixel size being $0.09 \mathrm{~mm}(\mathrm{H}) \times 0.09 \mathrm{~mm}(\mathrm{~V})$. Figure 4 shows the measured spectral data from the illumination display, with which the optimal optical elements in the system can be selected and ray-tracing model be built. According to Fig. 4, the major wavelength range for the display is $425 \mathrm{~nm}-650 \mathrm{~nm}$. A microscope objective (Mitutoyo Plan Apo 10× and NA 0.28) with long working distance is applied in the testing system, and the test object is a rough metal surface. 


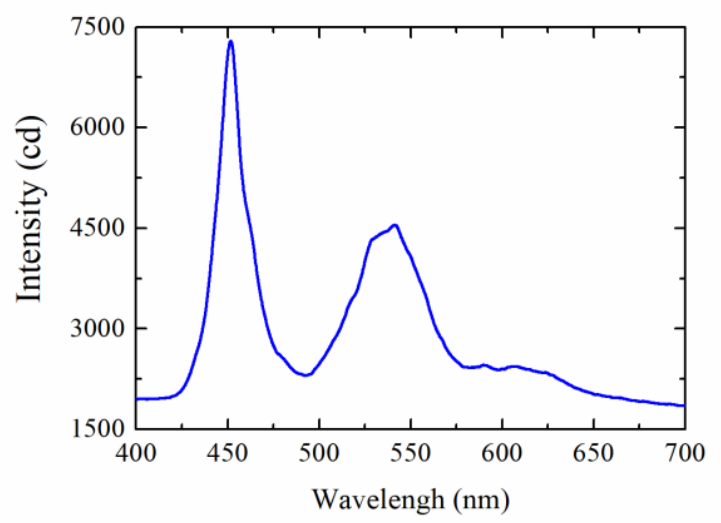

Fig. 4. Spectrum data for the LCD projection screen.

Figure 5(a) shows the acquired color-coded composite pattern after reflection at test surface. With the color separation, the composite fringe patterns in $x$ and $y$ directions extracted from the red and blue channels are shown in Figs. 5(b) and 5(c), respectively. Figures 5(d) and 5(e) are the demodulated fringe patterns in $x$ and $y$ directions. Using the extracted four-step phase shift fringes in $x$ and $y$ directions, the corresponding observed phase distributions $\left(\varphi_{x}, \varphi_{y}\right)$ can be obtained with the four-step phase shifting algorithm. According to Eq. (1), the slope data $\left(k_{x}, k_{y}\right)$ in $x$ and $y$ directions can be calculated to reconstruct the surface roughness with the Southwell integration algorithm. Figure 6 shows the surface roughness measured with the proposed transient microscopic testing method, whose peak-to-valley (PV) and root-meansquare (RMS) values are $1.7080 \mu \mathrm{m}$ and value $0.0954 \mu \mathrm{m}$, respectively.

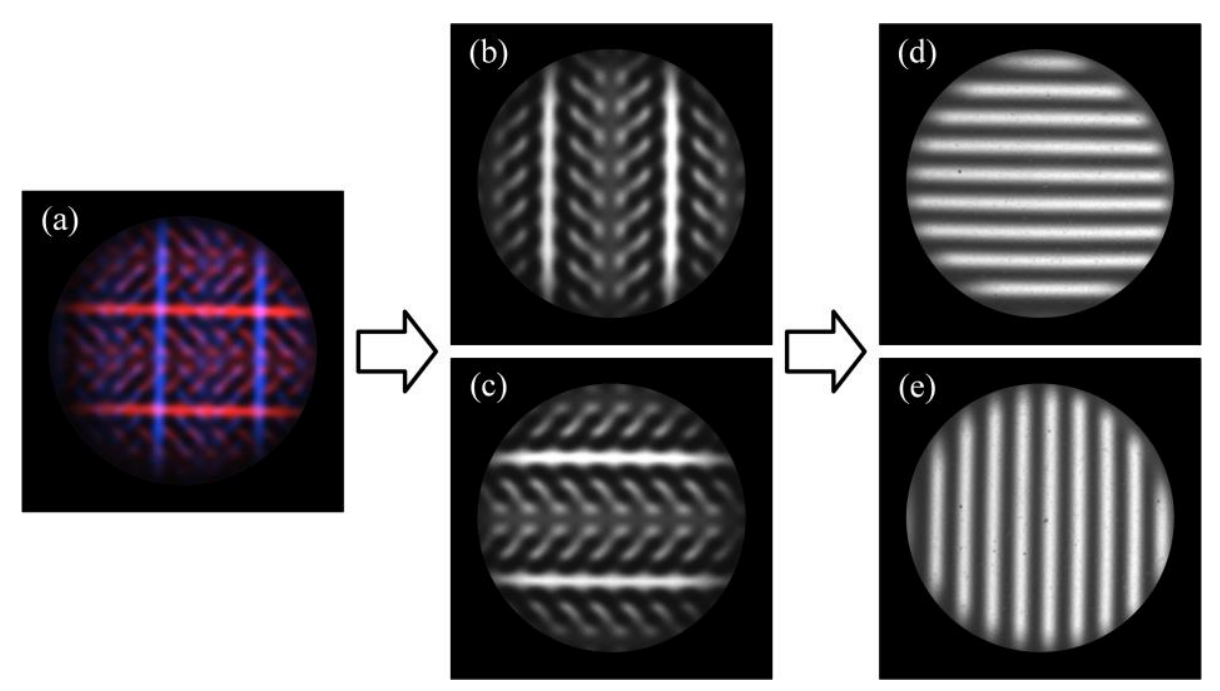

Fig. 5. Extraction of the color-coded composite pattern. (a) Acquired color-coded composite pattern; composite fringe patterns in (b) $x$ direction and (c) $y$ direction; Acquired fringes in (d) $x$ direction and (e) $y$ direction. 


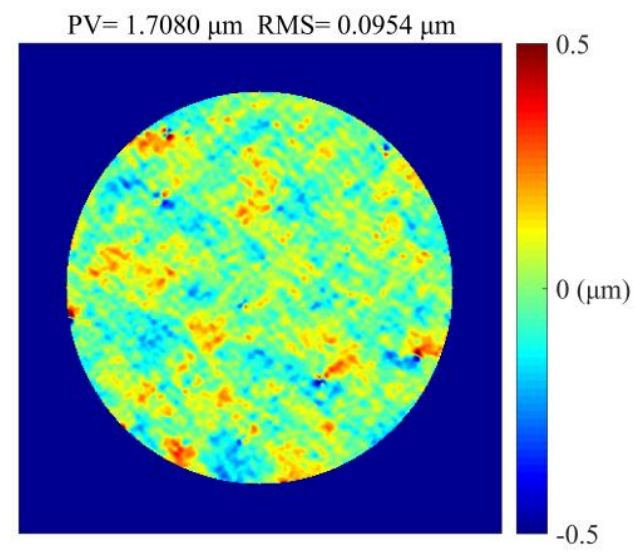

Fig. 6. Measured metal surface roughness with the proposed method.

\section{CONCLUSION}

In this paper, we put forward a transient microscopic method based on the deflectometry for the instantaneous testing of microstructures. In the deflectometry-based microscopic testing, the illumination module shares the same optical circuit with the detection path through a microscope objective. To realize transient measurement of microscopic surfaces, a color-coded composite pattern is designed as the projection pattern, in which the multiple phase-shifting fringes in $x$ and $y$ directions are integrated in a single-color image. With the application of the color-coded composite pattern, the transient microscopic testing can be achieved with a single shot. The experiment has been carried out to demonstrate the feasibility of the proposed testing method. The proposed testing method provides a feasible way to achieve the rapid, transient microscopic testing with large dynamic range, minimizing the environmental disturbance. In addition, the crosstalk among RGB channels and photon response nonuniformity could be largely avoided with the proposed transient method, and it does not require complex calibration.

\section{ACKNOWLEDGEMENTS}

The activities of this work are supported by National Natural Science Foundation of China (NSFC) (51775528), Guangxi Key Laboratory of Optoelectroric Information Processing (GD18205), Zhejiang Provincial Natural Science Foundation of China (LY17E050014), China Postdoctoral Science Foundation (2017M621928), Zhejiang Key Discipline of Instrument Science and Technology (JL150508).

\section{REFERENCES}

[1] Fukada, S., Fang, B. and Shigeno, A., "Experimental analysis and simulation of nonlinear microscopic behavior of ball," Precision Engineering 35(4), 650-668 (2011).

[2] He, P., Wang, F., Li, L., Georgiadis, K., Dambon, O., Klocke, K. and Yi, A. Y., "Development of a low cost high precision fabrication process for glass hybrid aspherical diffractive lenses," Journal of Optics 13(8), 085703 (2011).

[3] Hong, D., Lee, G., Jung, N. C. and Jeon, M., "Fast automated yeast cell counting algorithm using bright-field and fluorescence microscopic images," Biological Procedures Online 15(1), 1-8 (2013).

[4] Gao, W., Haitjema, H., Fang, F. Z., Leach, R. K., Cheung, C. F., Savio, E. and Linares, J. M., "On-machine and inprocess surface metrology for precision manufacturing," CIRP Annals 2024, 1-24 (2019).

[5] Zou, X., Zhao, X., Li, G., Li, Z. and Sun, T., "Non-contact on-machine measurement using a chromatic confocal probe for an ultra-precision turning machine," The International Journal of Advanced Manufacturing Technology 90, 2163-2172 (2017).

[6] Wang, D. and Liang, R., "Simultaneous polarization Mirau interferometer based on pixelated polarization camera," Optics Letters 41(1), 41-44 (2016).

[7] De Groot, P. J. and Biegen, J. F., "Interference microscope objectives for wide-field areal surface topography measurements," Optical Engineering 55(7), 074110 (2016). 
[8] Tian, X., Tu, X., Zhang, J., Spires, O., Brock, N., Pau, S. and Liang, R., "Snapshot multi-wavelength interference microscope," Optics Express 26(14), 18279-18291 (2018).

[9] Tian, X., Zhang, Y., Sohn, A., Spires, O. J. and Liang, R., "Dual-mode snapshot interferometric system for onmachine metrology," Optical Engineering 58(4), 044104 (2019).

[10] Wang, D., Gong, Z., Xu, P., Liang, R., Kong, M. and Zhao, J., "Accurate calibration of geometrical error in reflective surface testing based on reverse Hartmann test," Optics Express 26(7), 8113-8124 (2018).

[11] Wang, D., Zhang, S., Wu, R., Huang, C., Cheng, H. and Liang, R., "Computer-aided high-accuracy testing of reflective surface with reverse Hartmann test," Optics Express, 24(17), 19671-19681 (2016).

[12] Su, P., Wang, S., Khreishi, M., Wang, Y., Su, T., Zhou, P., Parks, R. E., Law, K., Rascon, M., Zobrist, T., Martin, H. and Burge, J. H., "SCOTS: A reverse Hartmann test with high dynamic range for giant Magellan telescope primary mirror segments," Proc. SPIE 8450, 84500W (2012).

[13] Dominguez, M., Wang, L., Su, P., Parks, R. E. and Burge, J. H., "Software configurable optical test for refractive optics," Proc. SPIE 8011, 80116Q (2011).

[14] Dominguez, M. Z., Armstrong, J., Su, P., Parks, R. E. and Burge, J. H., "SCOTS: a useful tool for specifying and testing optics in slope space," Proc. SPIE 8493, 84931D (2012).

[15] Häusler, G., Richter, G., Leitz, K. H. and Knauer, "Microdeflectometry-a novel tool to acquire three-dimensional microtopography with nanometer height resolution," Optics Letters 33(4), 396-398 (2008).

[16] Li, A., Peng, X., Yin, Y., Liu, X., Zhao, Q., Körner, K. and Osten, W, "Fringe projection based quantitative 3D microscopy," Optik 124(21), 5052-5056 (2013).

[17] Yin, Y., Wang, M., Gao, B. Z., Liu, X. and Peng, X., "Fringe projection 3D microscopy with the general imaging model," Optics Express 23(5), 6846-6857 (2015).

[18] Lu, S. and Hua, H., "Structured illumination assisted microdeflectometry with optical depth scanning capability," Optics Letter 41(17), 4114-4117 (2016).

[19] Selensnick, I. W. and Burrus, C. S., "Generalized digital Butterworth filter design," IEEE Transactions on Signal Processing 46(6), 1688-1689 (1998).

[20] Wang, D., Xu, P., Gong, Z., Xie, Z., Liang, R., Xu, X., Kong, M. and Zhao, J., "Transmitted wavefront testing with large dynamic range based on computer-aided deflectometry," Journal of Optics 20(6), 065705 (2018). 